\title{
LMVE at SemEval-2020 Task 4: Commonsense Validation and Explanation using Pretraining Language Model
}

\author{
Shilei Liu ${ }^{1}$, Yu Guo ${ }^{1}$, Bochao $\mathbf{L i}^{1}$ and Feiliang Ren ${ }^{12 *}$ \\ ${ }^{1}$ School of Computer Science and Engineering, Northeastern University \\ ${ }^{2}$ Key Laboratory of Data Analytics and Optimization for Smart \\ Industry (Northeastern University), Ministry of Education \\ $\{1901750,1871502,1901725\}$ estu.neu.edu.cn \\ renfeiliangeise.neu.edu.cn
}

\begin{abstract}
This paper describes our submission ${ }^{1}$ to subtask a and b of SemEval-2020 Task 4. For subtask a, we use a ALBERT based model with improved input form to pick out the common sense statement from two statement candidates. For subtask b, we use a multiple choice model enhanced by hint sentence mechanism to select the reason from given options about why a statement is against common sense. Besides, we propose a novel transfer learning strategy between subtasks which help improve the performance. The accuracy scores of our system are 95.6 / 94.9 on official test set $^{2}$ and rank $7^{\text {th }} / 2^{\text {nd }}$ on Post-Evaluation leaderboard.
\end{abstract}

\section{Introduction}

Common sense verification and explanation is an important and challenging task in artificial intelligence and natural language processing. This is a simple task for human beings, because human beings can make full use of external knowledge accumulated in their daily lives. However, common sense verification and reasoning is difficult for machines. According to Wang (2019), even some state-of-the-art language models such as ELMO(Peters et al., 2018) and BERT(Devlin et al., 2019) have very poor performance. So it is crucial to integrate the ability of commonsense-aware to natural language understanding model(Davis, 2017).

SemEval-2020 task4(Wang et al., 2020) aims to improve the ability of common sense judgment for model, and we participated in two subtasks of this task. The dataset of SemEval-2020 task4 named ComVE. Each instance in ComVE is composed of 5 sentences $\left\langle s_{1}, s_{2}, o_{1}, o_{2}, o_{3}\right\rangle . s_{1}$ and $s_{2}$ will be used for subtask a, and $s_{1}$ or $s_{2}$ with $\left\langle o_{1}, o_{2}, o_{3}\right\rangle$ will be used for subtask b.

Subtask a(also known as Sen-Making task) aims to test a model's ability of commonsense validation. Specifically, given two statements $\left\langle s_{1}, s_{2}\right\rangle$ whose lexical and syntactic are similar, the object of SenMaking model is to determine which statement is common sense(compared to another one). For example, $s_{1}$ is put the elephant in the refrigerator and $s_{2}$ is put the turkey in the refrigerator, a good model needs to judge that the latter is more common sense.

Subtask b(also known as Explanation task) is a multiple choice task that aims to find the key reason why a given statement does not make sense. For example, given a sentence that violates common sense with three options $\left\langle s, o_{1}, o_{2}, o_{3}\right\rangle$, where $s$ is he put an elephant into the fridge, $o_{1}$ is an elephant is much bigger than a fridge, $o_{2}$ is elephants are usually gray while fridges are usually white, and $o_{3}$ is an elephant cannot eat a fridge, the model needs to judge that $o_{1}$ is the correct option.

The official baseline of Sen-Making use a pretraining language model(PLM) to dynamic encoding the two input sentence, and use a simple full connection neural network to calculate the perplexities respectively, and choosing the one with lower scores as the correct one. We believe that the baseline method treats two sentences independently and ignores the inner relationship between the two sentences, so we propose a novel model structure that fully considers the interaction between statements.

\footnotetext{
${ }^{*}$ Corresponding author.

${ }^{1}$ Our team name on leaderboard is NEUKG, and the first three authors contributed equally to this work.

${ }^{2}$ These scores were obtained during the Post-Evaluation phase, and our scores in the Evaluation phase are 90.04 / 93.8. This work is licensed under a Creative Commons Attribution 4.0 International Licence. Licence details: http:// creativecommons.org/licenses/by/4.0/.
} 
The official baseline of Explanation treats the task as BERT-like multiple choice task(Devlin et al., 2019). We think that the baseline model doesn't make full use of the input data. So we design a structure to incorporate another statement that is common sense to existing model.

In addition, we believe that fine-tuning on similar subtask can improve the performance of the current subtask because there are many commonalities between the two subtasks, so we propose a novel transfer learning mechanism between Sen-Making and Explanation.

The proposed system named LMVE, it is a neural network model(includes two sub-modules to solve both subtask $a$ and $b$ ) bases on large scale pretraining language model.

Our contributions are as follows:

- First, we propose subtask level transfer learning that help share information between subtasks.

- Second, we propose a novel structure to calculate the perplexity of sentence, which takes into account the interaction between sentences in a pair.

- Third, we propose the hint sentence mechanism that will help improve the performance of multiple choice task.(subtask b).

\section{System Description}

We consider our model for both Sen-Making and Explanation as two parts: encoder and decoder. Encoder is mainly used for getting the contextual representation of input sentence tokens. In recent years, some pretraining language models including BERT(Devlin et al., 2019), RoBERTa(Liu et al., 2019) and ALBERT(Lan et al., 2020) have been proven beneficial for many natural language processing (NLP) tasks(Rajpurkar et al., 2016; Bowman et al., 2015). These pretrained models have learned general-purpose language representations on a large amount of unlabeled data, therefore, adapting these models to the downstream tasks can bring a good initialization for parameters and avoid training from scratch(Xu et al., 2020). So we tried some popular PLMs as encoders. Decoder consists of several simple linear layers whose number of parameters are far less than encoder, and the role of decoder is to fuse the output of encoder and predict the answer.

\subsection{LMVE for Sen-Making Task}

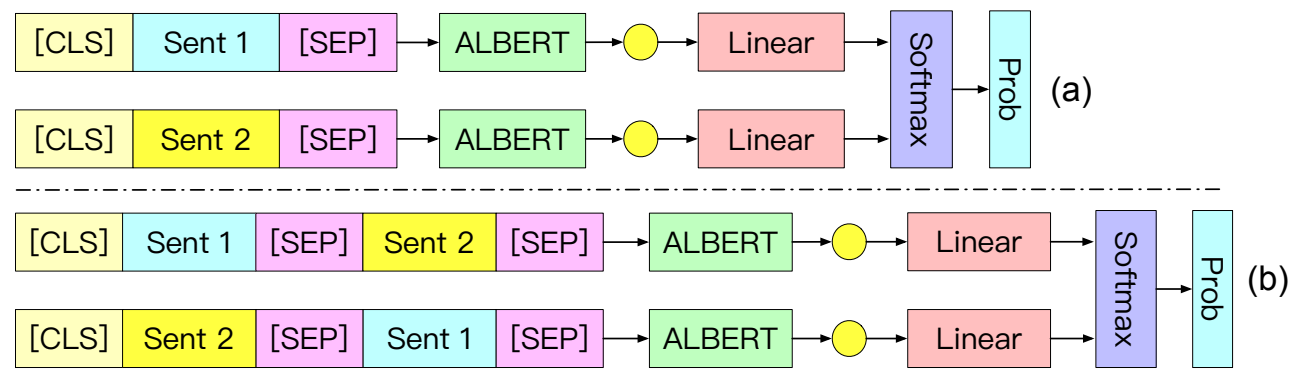

Figure 1: The model architecture for Sen-Making task, (a) is official baseline and (b) is ours. The yellow point denotes the vector representation of the output sequence(same as below).

Figure 1(a) is the official baseline model(Wang et al., 2019), which regards two sentences as independent individuals. But in ComVE, there are certain similarities(lexical and grammatical) between the two statements, so we think that the interaction between the two sentences is helpful to improve the performance of the model. Figure 1(b) gives an overview of our model for Sen-Making task which is mainly composed of three modules including token encoding, feature fusion and answer prediction.

Encoding: Let $\left\{x_{0}^{1}, \ldots, x_{p}^{1}\right\}$ and $\left\{x_{0}^{2}, \ldots, x_{q}^{2}\right\}$ represent one-hot vectors for the first sentence and the second sentence in an instance, we first concatenate them and add some special tokens like Figure 1(b), then we will get two sequences $\left\{x_{[C L S]}, x_{0}^{1}, \ldots, x_{p}^{1}, x_{[S E P]}, x_{0}^{2}, \ldots, x_{q}^{2}, x_{[S E P]}\right\}$ and 
$\left\{x_{[C L S]}, x_{0}^{2}, \ldots, x_{q}^{2}, x_{[S E P]}, x_{0}^{1}, \ldots, x_{p}^{1}, x_{[S E P]}\right\}$. The two sequences will be fed into ALBERT respectively. We use $U^{i} \in R^{d \times n}$ and $V^{i} \in R^{d \times n}$ denote outputs of $i$-th transformer block in ALBERT, where $d$ is the hidden size of model, $n$ is the sequence length, and $i \in\{0, \ldots, L-1\}$.

Fusion: Some pretraining language model(BERT et al.) usually take the first token (corresponds to [CLS ] ) of the output of last transformer block as the representation of a sequence, but we use the weighted sum of the representation of first token in all transformer block outputs as the final representation ${ }^{3}$. The following equations describe the process of fusion:

$$
\begin{aligned}
r_{i}^{0} & =U_{0}^{i} \quad r_{i}^{1}=V_{0}^{i} \\
\alpha_{i} & =\frac{\exp \left(\omega_{\mathbf{i}}\right)}{\sum_{j} \exp \left(\omega_{\mathbf{j}}\right)} \\
x_{k} & =\sum_{i=0}^{L-1} \alpha_{i} r_{i}^{k}
\end{aligned}
$$

where $\omega \in \mathbb{R}^{d}$ is a trainable parameter and $i \in[0, L-1]$. We can regard $x_{k}$ as the representation of $k$-th statement $(k \in\{0,1\})$.

Answer Prediction: This module maps the output of the fusion layer to a probability distribution of answer. Given $\mathbf{w} \in \mathbb{R}^{d}$ and $b \in \mathbb{R}$ as learnable parameters, it calculates the answer possibility as

$$
p(k)=\operatorname{softmax}\left(\mathbf{w} \boldsymbol{x}_{k}+b\right)
$$

We define the training loss as cross entropy loss function:

$$
\mathcal{L}_{a}=-\frac{1}{N} \sum_{i=1}^{N} p\left(y_{i}\right)
$$

where $N$ is the number of samples in the dataset and $y_{i} \in\{0,1\}$.

\subsection{Hint Sentence mechanism}

Before formally introducing our model for Explanation task, let's first introduce the hint sentence mechanism.

In official baseline(Wang et al., 2019), the against common sense statement will be concatenated with three options respectively and fed into the model. We believe that this form of input does not make full use of the data in the ComVE. Specifically, for an instance $\left\langle s_{1}, s_{2}, o_{1}, o_{2}, o_{3}\right\rangle$ in ComVE, it is assumed that $s_{1}$ does not conform to common sense, then $\left\langle s_{2}, o_{1}, o_{2}, o_{3}\right\rangle$ will be used to train the baseline model or to predict answer. However, in this process, $s_{1}$ was abandoned. We believe that another common sense statement $\left(s_{1}\right)$ in statement pair contains some useful information, and should be incorporated into our model.

So we propose hint sentence mechanism: A hint sentence is common sense and its lexical and syntactic are similar to the given against common sense statement and they differ by only few words. In other words, we call the another sentence in the sentence pair a hint sentence.

The process of how the hint sentence is integrated into the existing model can be referred to next section and Figure 2. The results of ablation experiment( $\mathrm{Sec} 3.6)$ show that hint sentence mechanism can greatly improve the performance of our model for Explanation task.

\subsection{LVME for Explanation Task}

Figure 2 gives an overview of our model for Explanation task. it also has three modules.

Let $\left\{s_{0}, \ldots, s_{p}\right\},\left\{h_{0}, \ldots, h_{q}\right\}$ and $\left\{o_{0}^{i}, \ldots, o_{r_{i}}^{i}\right\}$ represent one-hot vectors for the input statement, hint sentence and $i$-th option in an instance, where $i \in\{0,1,2\}$ and $p, q$ and $r$ is the length of them, we first concatenate them and add some special tokens like Figure 2, then we will get three sequences

\footnotetext{
${ }^{3}$ Subsequent experimental results show that the performance using the last 4 layers is the best.
} 


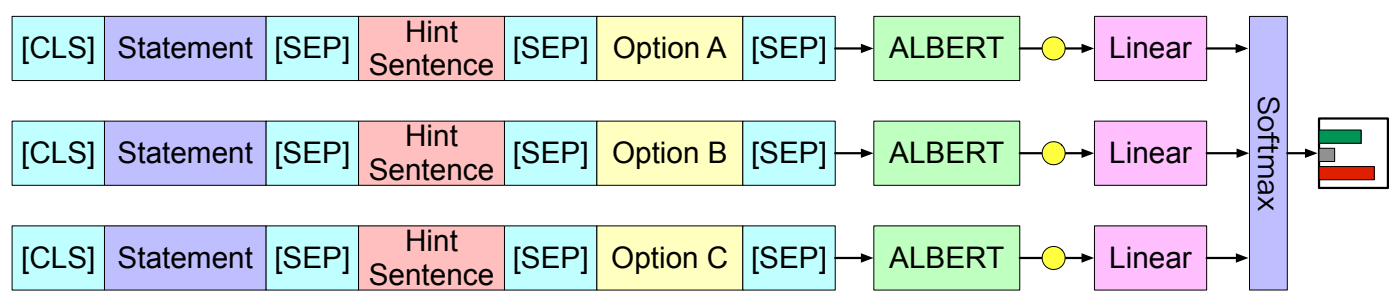

Figure 2: The model architecture for Explanation task.

$\left\{x_{[C L S]}, s_{0}, \ldots, s_{p}, x_{[S E P]}, h_{0}, \ldots, h_{q}, x_{[S E P]}, o_{0}^{i}, \ldots, o_{r_{i}}^{i}, x_{[S E P]}\right\}$. Then the three sequences will be fed into ALBERT respectively.

Similar to last sub-section, each sequence will get a representation vector after fusion, and then the three representation vector will pass a linear layer like Equation 4 to calculate the probability distributions of answer.

We define training loss as

$$
\mathcal{L}_{b}=-\frac{1}{N} \sum_{i=1}^{N} p\left(y_{i}\right)
$$

where $N$ is the number of samples in the dataset and $y_{i} \in\{0,1,2\}$ is true label.

\subsection{Subtask Level Transfer Learning}

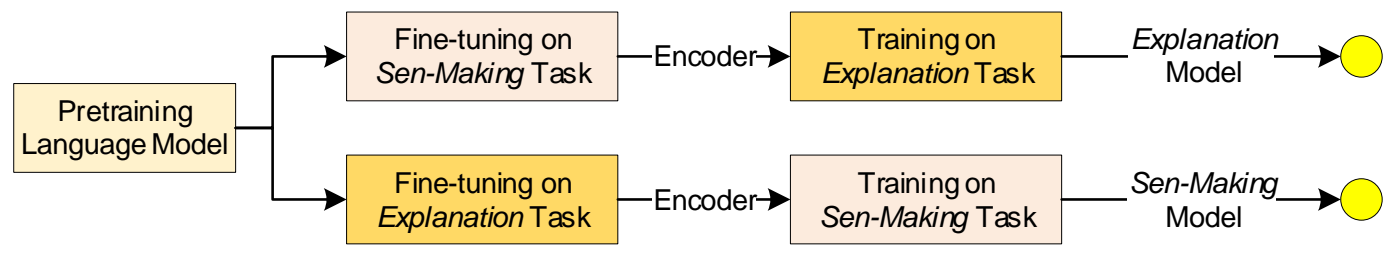

Figure 3: The process of subtask level transfer learning.

Transfer learning is a research problem in machine learning that focuses on storing knowledge gained while solving one problem and applying it to a different but related problem. PLM is a typical example of transfer learning and we call it task level transfer learning.

Sen-Making task and Explanation task are both generalized multiple choice tasks, and there is an association between the input data for them, so we believe that in SemEval-2020 Task 4, fine-tuning on similar subtask can improve the performance of the current subtask.

Subtask level transfer learning refers to use the encoder after fine-tuning on subtask a(Sen-Making) to train subtask b(Explanation) and vice versa. The process of Subtask level transfer learning are shown in Figure 3.

\section{Experiments and Analysis}

\subsection{Dataset}

ComVE include 10000 samples in train set and 1000 samples in dev/test set for both Sen-Making and Explanation task. The average length of two statements in the Sen-Making task are both 8.26, exactly the same. The average length of true reasons is 7.63 in Explanation task.

It should be noted that in SemEval-2020 Task 4, the test set of Explanation task is issued only after Sen-Making task is completed, so it is impossible to use the test set of Explanation task to reverse deduce the answer of subtask a test set.

\subsection{Baseline}

To verify the effectiveness of our model, we used ALBERT to replace the BERT in the official baseline, leaving the rest unchanged. We do not perform subtask level transfer learning (Sec 2.4) on them. 

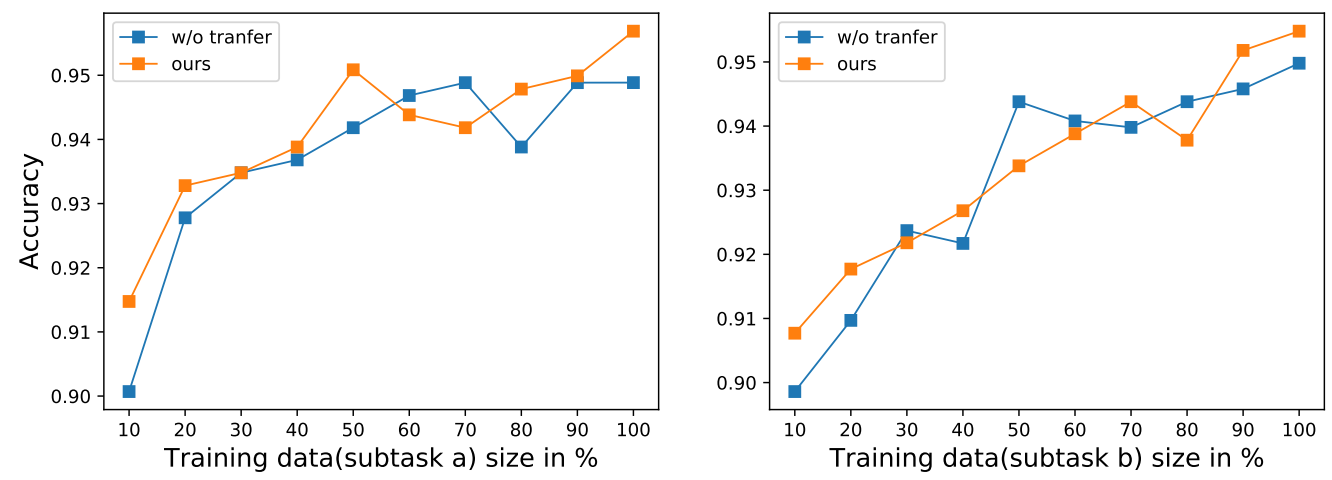

Figure 4: Learning curve on the training dataset.

\subsection{Preprocessing}

Data Augmentation: To enhance the robustness of our model, we use Google Sheets ${ }^{4}$ to perform back translation technology on original texts to get augmented texts. Specifically, given a training sample $\left\langle s_{1}, s_{2}, o_{1}, o_{2}, o_{3}\right\rangle$ we first translate the original statements $s_{1}, s_{2}$ to French and then translate them back to English (denoted as $\hat{s_{1}}, \hat{s_{2}}$ ). $\left\langle\hat{s_{1}}, \hat{s_{2}}, o_{1}, o_{2}, o_{3}\right\rangle$ will add to training dataset as a new sample. the size of the dataset has doubled after augmentation.

Tokenization: We employ the tokenizer that comes with the HuggingFace(Wolf et al., 2019) PyTorch implementation of ALBERT. The tokenizer lowercases the input and applies the SentencePiece encoding(Kudo, 2018) to split input words into most frequent subwords present in the pre-training corpus. Non-English characters will be removed.

\subsection{Implementation Details}

We use the Transformers ${ }^{5}$ toolkit to implemented our model and tune the hyper-parameters according to validation performance on the development set. The hidden size is equal to the corresponding PLM. To train our model, we employ the AdamW algorithm(Loshchilov and Hutter, 2019) with the initial learning rate as $2 \mathrm{e}-5$ and the mini-batch size as 48 .

We also prepared an ensemble model consisting of 7 models for Sen-Making task and 19 for Explanation task with different hyperparameter settings and random seeds. We used majority voting strategy to fuse the candidate predictions of different models together.

\begin{tabular}{|c|c|c|c|}
\hline Model & Params & Sen-Making & Explanation \\
\hline Random & - & 49.52 & 32.77 \\
\hline $\mathrm{BERT}_{\text {base }}($ Devlin et al., 2019) & $117 \mathrm{M}$ & 88.56 & 85.32 \\
\hline $\mathrm{BERT}_{\text {large }}($ Devlin et al., 2019) & $340 \mathrm{M}$ & 86.55 & 90.12 \\
\hline XLNet(Yang et al., 2019) & $340 \mathrm{M}$ & 90.33 & 91.07 \\
\hline SpanBERT(Joshi et al., 2019) & $340 \mathrm{M}$ & 89.46 & 90.47 \\
\hline RoBERTa(Liu et al., 2019) & $355 \mathrm{M}$ & 93.56 & 92.37 \\
\hline $\operatorname{ALBERT}_{\text {base }}($ Lan et al., 2020) & $12 \mathrm{M}$ & 86.63 & 84.37 \\
\hline $\operatorname{ALBERT}_{\text {large }}($ Lan et al., 2020) & $18 \mathrm{M}$ & 88.01 & 89.72 \\
\hline ALBERT $_{\text {xlarge }}($ Lan et al., 2020) & $60 \mathrm{M}$ & 92.03 & 92.45 \\
\hline Ours(ALBERT $\left.{ }_{\text {xxlarge }}\right)$ & $235 \mathrm{M}$ & 95.68 & 95.48 \\
\hline Our-ensemble & - & 95.91 & 96.39 \\
\hline
\end{tabular}

Table 1: Performance with different encoder.

\footnotetext{
${ }^{4}$ https://www.google.com/sheets/about

${ }^{5}$ https://github.com/huggingface/transformers
} 


\subsection{Main Result}

The result of our model for subtask $a$ and subtask $b$ are summarized in Table 1. We have tried different pretraining language model as our encoder, and found that ALBERT based model achieves the state-ofthe-art performance.

Figure 4 shows a learning curve computed over the provided training data with testing against the development set, and we can see that in the case of low-resource (only use 10\%-20\% training data of target task), the performance of introducing subtask level transfer learning is significantly higher than original implementation.

\begin{tabular}{lll}
\hline Sen-Making Model & Acc & $\Delta_{a}$ \\
\hline Our-single & 95.68 & - \\
w/o method b $^{\dagger}$ & 94.88 & 0.80 \\
w/o data augmentation & 95.43 & 0.25 \\
w/o weighted sum fusion & 95.32 & 0.36 \\
w/o subtask level transfer & 94.85 & 0.83 \\
Baseline & 93.24 & 2.44 \\
\hline
\end{tabular}

\begin{tabular}{lll}
\hline Explanation Model & Acc & $\Delta_{b}$ \\
\hline Our-single & 95.48 & - \\
w/o hint sentence & 93.47 & 2.01 \\
w/o data augmentation & 95.39 & 0.09 \\
w/o weighted sum fusion & 95.11 & 0.37 \\
w/o subtask level transfer & 94.98 & 0.50 \\
Baseline & 93.12 & 2.36 \\
\hline
\end{tabular}

Table 2: Ablation study on model components. $\dagger$ means we use the model structure as Figure 1(a) and Baseline means the model in Sec 3.2.

\subsection{Ablation Study}

To get better insight into our model architecture, we conduct an ablation study on dev set of ComVE, and the results are shown in Table 2.

From the results we can see that subtask level transfer learning has a relatively large contribution for both subtask $a$ and $b$, which confirms our hypothesis that fine-tuning on similar task can improve the performance of the current task. Data augmentation and weighted sum fusion also have minor contributions due to the more robust dataset and more robust model.

For subtask a, we can see that compared with baseline method(Figure 1(a)), concatenating another sentence as input can have a higher performance. We speculate that the reason is traditional method treat two statements as independent individuals, and our method takes into account the inherent connection between the two statements.

For subtask $\mathrm{b}$, we can see from Table 2 that hint sentence makes a great contribution the overall improvement. We think the reason is a common sense statement with a similar grammar and syntax does help the model to determine why the input sentence is against common sense.

\section{Conclusions}

This paper introduces our system for commonsense validation and explanation. For Sen-Making task, we use a novel pretraining language model based architecture to pick out one of the two given statements that is against common sense. For Explanation task, we use a hint sentence mechanism to improve the performance greatly. In addition, we propose a subtask level transfer learning to share information between subtasks.

As future work, we plan to integrate the external knowledge base(such as ConceptNet ${ }^{6}$ ) into commonsense inference.

\section{Acknowledgements}

We thank the reviewers for their helpful comments. This work is supported by the National Natural Science Foundation of China (No. 61572120) and the Fundamental Research Funds for the Central Universities (No.N181602013).

\footnotetext{
${ }^{6} \mathrm{http}: / / \mathrm{www}$. conceptnet.io/
} 


\section{References}

Samuel R. Bowman, Gabor Angeli, Christopher Potts, and Christopher D. Manning. 2015. A large annotated corpus for learning natural language inference. In Lluís Màrquez, Chris Callison-Burch, Jian Su, Daniele Pighin, and Yuval Marton, editors, Proceedings of the 2015 Conference on Empirical Methods in Natural Language Processing, EMNLP 2015, Lisbon, Portugal, September 17-21, 2015, pages 632-642. The Association for Computational Linguistics.

Ernest Davis. 2017. Logical formalizations of commonsense reasoning: A survey. J. Artif. Intell. Res., 59:651723.

Jacob Devlin, Ming-Wei Chang, Kenton Lee, and Kristina Toutanova. 2019. BERT: Pre-training of Deep Bidirectional Transformers for Language Understanding. In Proceedings of the 2019 Conference of the North American Chapter of the Association for Computational Linguistics: Human Language Technologies, Volume 1 (Long and Short Papers), pages 4171-4186, Minneapolis, Minnesota. Association for Computational Linguistics.

Mandar Joshi, Danqi Chen, Yinhan Liu, Daniel S. Weld, Luke Zettlemoyer, and Omer Levy. 2019. Spanbert: Improving pre-training by representing and predicting spans. CoRR, abs/1907.10529.

Taku Kudo. 2018. Subword regularization: Improving neural network translation models with multiple subword candidates. In Iryna Gurevych and Yusuke Miyao, editors, Proceedings of the 56th Annual Meeting of the Association for Computational Linguistics, ACL 2018, Melbourne, Australia, July 15-20, 2018, Volume 1: Long Papers, pages 66-75. Association for Computational Linguistics.

Zhenzhong Lan, Mingda Chen, Sebastian Goodman, Kevin Gimpel, Piyush Sharma, and Radu Soricut. 2020. \{ALBERT\}: A Lite $\{$ BERT $\}$ for Self-supervised Learning of Language Representations. In International Conference on Learning Representations.

Yinhan Liu, Myle Ott, Naman Goyal, Jingfei Du, Mandar Joshi, Danqi Chen, Omer Levy, Mike Lewis, Luke Zettlemoyer, and Veselin Stoyanov. 2019. Roberta: A robustly optimized BERT pretraining approach. CoRR, abs/1907.11692.

Ilya Loshchilov and Frank Hutter. 2019. Decoupled weight decay regularization. In 7th International Conference on Learning Representations, ICLR 2019, New Orleans, LA, USA, May 6-9, 2019. OpenReview.net.

Matthew Peters, Mark Neumann, Mohit Iyyer, Matt Gardner, Christopher Clark, Kenton Lee, and Luke Zettlemoyer. 2018. Deep Contextualized Word Representations. In Proceedings of the 2018 Conference of the North American Chapter of the Association for Computational Linguistics: Human Language Technologies, Volume 1 (Long Papers), pages 2227-2237, New Orleans, Louisiana. Association for Computational Linguistics.

Pranav Rajpurkar, Jian Zhang, Konstantin Lopyrev, and Percy Liang. 2016. Squad: 100, 000+ questions for machine comprehension of text. In Jian Su, Xavier Carreras, and Kevin Duh, editors, Proceedings of the 2016 Conference on Empirical Methods in Natural Language Processing, EMNLP 2016, Austin, Texas, USA, November 1-4, 2016, pages 2383-2392. The Association for Computational Linguistics.

Cunxiang Wang, Shuailong Liang, Yue Zhang, Xiaonan Li, and Tian Gao. 2019. Does it make sense? and why? A pilot study for sense making and explanation. In Anna Korhonen, David R. Traum, and Lluís Màrquez, editors, Proceedings of the 57th Conference of the Association for Computational Linguistics, ACL 2019, Florence, Italy, July 28-August 2, 2019, Volume 1: Long Papers, pages 4020-4026. Association for Computational Linguistics.

Cunxiang Wang, Shuailong Liang, Yili Jin, Yilong Wang, Xiaodan Zhu, and Yue Zhang. 2020. SemEval-2020 task 4: Commonsense validation and explanation. In Proceedings of The 14th International Workshop on Semantic Evaluation. Association for Computational Linguistics.

Thomas Wolf, Lysandre Debut, Victor Sanh, Julien Chaumond, Clement Delangue, Anthony Moi, Pierric Cistac, Tim Rault, R'emi Louf, Morgan Funtowicz, and Jamie Brew. 2019. Huggingface's transformers: State-of-theart natural language processing. ArXiv, abs/1910.03771.

Yige Xu, Xipeng Qiu, Ligao Zhou, and Xuanjing Huang. 2020. Improving BERT Fine-Tuning via Self-Ensemble and Self-Distillation. arXiv:2002.10345 [cs], February. arXiv: 2002.10345.

Zhilin Yang, Zihang Dai, Yiming Yang, Jaime G. Carbonell, Ruslan Salakhutdinov, and Quoc V. Le. 2019. XLNet: Generalized Autoregressive Pretraining for Language Understanding. In Advances in Neural Information Processing Systems 32: Annual Conference on Neural Information Processing Systems 2019, NeurIPS 2019, 8-14 December 2019, Vancouver, BC, Canada, pages 5754-5764. 